\title{
Friendly Object Tracking and Foreign Object Detection and Localization with an SDAC Wireless Sensor Network
}

\author{
Douglas Stark and Jesse Davis \\ Sandia National Labs, Livermore, California, USA \\ \{dpstark, jzdavis\}@sandia.gov
}

\begin{abstract}
This paper presents a proof-of-concept wireless sensor network for detecting and tracking friendly objects and detecting and localizing foreign objects. The network incorporates a novel sensor network methodology to $\underline{S}$ ense its surroundings, Decide in-network what the situation in its surroundings is, Act on this decision, and Communicate its results to a user (SDAC). The system also performs in-network data fusion and data reduction and demonstrates the use of an adaptable hardware and software platform. The end user is presented with a Geographical Information Systems (GIS) viewer showing when and where objects are detected. The viewer is able to display tracking information about friendly objects by searching a database for events involving specific friendly objects.
\end{abstract}

\section{Introduction}

The combination of low cost, low power electronics with power aware software techniques, long life batteries, and low power, short-range radios has created the recently emerging technology of wireless sensor networks. The general thrust behind this technology is to provide ubiquitous, real-time, and intelligent data collection and information generation over physically widespread or complex spaces. Enabled with wireless transceivers and microprocessors, the nodes that make up the network may communicate and collaborate amongst themselves to create knowledge of the current situation in their surrounding environment rather than simply being data sources. Allowing the nodes to perform in-network distributed computation removes the strain on a user of having to sift through hundreds or thousands of data samples, and instead automates the knowledge generation process by intelligent in-network data fusion and data reduction. Additionally, performing in-network data reduction increases the lifetime of the sensor network. By far the largest consumer of a node's power budget is wireless communication, and by reducing the amount of raw data that must be sent to the user through the network, the overall wireless communications load on the network is reduced, thereby extending system lifetime [1]. Instead of relying on information-poor single-point sensing, or inefficient, costly, or dangerous human data collection, sensor networks also provide a revolutionary tool for fine grained, low cost, and adaptable sensing. Another primary benefit to distributing the sensing across an adaptable network is that it creates a more robust solution to the standard single-point-of-failure single-point sensors. There are many potential industrial, commercial, consumer, and military applications of sensor networks, but in general they can be used to facilitate efficiency, allow wide-spread monitoring and feedback, greatly reduce a need for human involvement in hazardous areas, and provide smart environments that assist their inhabitants.

Of particular interest to several applications is the problem of friendly object (human or material) tracking and foreign object detection and localization. For example, in a sociological application, an MIT Media Lab project is monitoring the movement of a family around an apartment in order to develop more efficient architecture designs [2]. The foreign object detection and localization in this application might represent an alarm system. Another example in a completely different domain is in automated factories. Collaborative mobile robots must know where each other are as well as any unknown foreign objects that must either be avoided or removed [3]. Finally, several security applications require the tracking and monitoring of friendly objects and the simultaneous localization of non-friendly intrusion. These security applications may be military, industrial, or commercial including border security, facility and factory protection, or farm and livestock monitoring and protection.

In order to explore the problems and concepts associated with object tracking in a wireless sensor network, the Embedded Reasoning Institute at Sandia National Laboratories in Livermore, California was tasked with creating a proof-of-concept wireless sensor network incorporating a novel sensor network methodology to Sense its surroundings, Decide in-network what the 
situation in its surroundings is, Act on this decision, and Communicate its results to a user (SDAC). The system was also required to perform in-network sensor fusion and data reduction on a computationally limited system, demonstrate the concept of the "network-as-a-sensor," and demonstrate the use of an adaptable hardware and software platform.

\section{Approach}

The problem of object tracking has been explored in a variety of contexts and on a variety of platforms. A cursory examination of existing object tracking systems reveals that many systems rely on cameras, computationally intensive computer vision algorithms, and easily identifiable objects. The largest difference that separates most of these previous approaches from the one described here is that the wireless sensor network nodes used for implementation are a computationally limited platform, and previous approaches have not been specifically resource constrained. While using low power eight- or sixteen-bit processors will result in a low-cost, long-life system, it also eliminates several possible implementations.

The Robotics Institute at Carnegie Mellon developed a robotic soccer team that uses a distributed sensor fusion algorithm to estimate the position of the soccer ball on the playing field. Applications such as robotic soccer require multiple mobile robots to know the location of a known object (the soccer ball) and each other. Implemented in C and Java, and running on a Pentium 266, the Carnegie Mellon algorithms relied on the known locations of the robots and two-dimensional probabilistic representations of the location of the soccer ball. In this case, each robot was able to share its perceived location of the ball with the other robots using only the location of the ball and twodimensional standard deviation of a Gaussian distribution at that location [3].

A similar level of data reduction was used in another system, built at Utsunomiya University in Utsunomiya, Japan, which was capable of performing multi-target tracking using distributed computing. In this case, the estimated locations of targets were computed by knowing the location of target imagers and bearing angles and ranges to the target [4]. While this level of data reduction and data fusion would be acceptable for a computationally limited sensor network, the camera interface and vision algorithm used as to detect the target would be nearly impossible to implement on computationally constrained systems. Similar conclusions can be reached for many other robot- or computer-based systems $[5,6]$.
One aspect of several systems that can be incorporated into a sensor network is the concept that target objects should be easily identifiable in some way. The robotic soccer team used a ball that was easily distinguishable from the playing field. Another robotics application optically identified brightly colored cylinders since they could be readily picked out from the background [5]. The same principle can be incorporated into a sensor network by equipping known objects with an RF transponder or RFID tag. This not only allows known objects to be easily distinguished from foreign objects, but also allows for tracking of specifically identified known objects. If an object is detected by an array of environmental sensors, it can be queried using RF. Foreign object are assumed to be unequipped with an appropriate RF transponder or RFID tag, and therefore unable to respond to the query, while known objects will respond and be identified.

Detecting objects as they move through an area could be accomplished through a variety of methods. The optical method has been explored thoroughly in the previously mentioned robotics applications and is generally not suitable for use in a low-cost, low-power sensor network using computationally limited hardware. In sensor network application developed at the University of Massachusetts, sensor nodes equipped with radar sensors collaborated to detect and track a target in real time [7]. However, the radar signals required substantial computing power to process and correlate. Similar sensors, such as infrared or ultrasonic sensors, could be used to provide similar though less precise information and are available in packages that do not require complicated analysis. The approach taken in this paper is to use simple in-network data fusion rules with passive infrared (PIR), acoustic, and seismic trigger sensors for object detection. Friendly object identification and tracking is accomplished by equipping friendly objects with a two-way RF transponder programmed with a unique identification number. This allows for an extremely simple system algorithmically, and correspondingly an extremely low power and low cost implementation.

\section{Implementation}

The Embedded Reasoning Institute at Sandia National Labs in Livermore, California has created a wireless sensor network system using distributed geophones, microphones, passive infrared (PIR) sensors (motion detectors), and GPS receivers in combination with twoway object-worn RF transponders to detect, track, and identify friendly objects and distinguish these from nonfriendly intrusions in an outside environment. The system 
is based on the SDAC sensor network methodology. Furthermore, built on top of proprietary networking protocols, innovative data fusion concepts are utilized to combine time correlated orthogonal sensor readings from several wirelessly connected nodes to effectively decrease the probability of false alarm and increase the probability of detection of events in the surrounding environment. The in-network data fusion also provides a data reduction so that users are only exposed to when and where friendly and foreign objects are located. This protection of the user from the complexities of the raw sensor data and network and node implementation details serves to increase user friendliness and realize the vision of the "network-as-asensor". This vision refers to the concept that the network essentially becomes a meta-sensor that senses friendly and non-friendly objects instead of the underlying seismic, acoustic, or motion time signatures.

In addition to the object localization application, a unique modular architecture was developed as a prototyping platform for this and future applications. The modularity allows extensibility and upgradeability more than a standard centralized system would because new sensing, processing, and communication components can simply be added and replaced without requiring any fundamental system re-design [8]. The modularity also provides a natural architecture for decentralizing the system operations. Instead of running data collection, processing, and communication tasks from a single schedule on a central processor, each resource-specific module has a processor that operates in parallel to the other modules to carry out its own resource-specific system function. This allows infrequently used high power system components, such as a general-purpose processor or imager, to remain in a low power sleep mode for the majority of the node's lifetime. The decentralized control thus provides a means for drastically reducing the total system power for a wide variety of applications [9]. Furthermore, this modularity leads to a more robust system since if one of the modules fails, the other parts of the system will still function. The modularity is enabled using an $\mathrm{I} 2 \mathrm{C}$ bus and a proprietary messaging structure. Each module is approximately 1.75 " by 2.20 ", and uses two 40-pin, 0.3" spacing bus connectors on each side of the board to stabilize and assemble a full node stack.

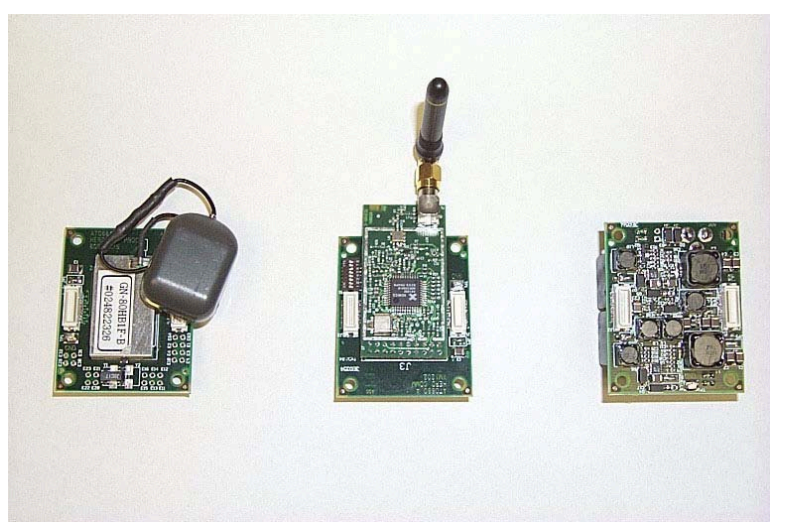

Figure 1: SDAC modules. Left to right: controller networking module, and power supply module.

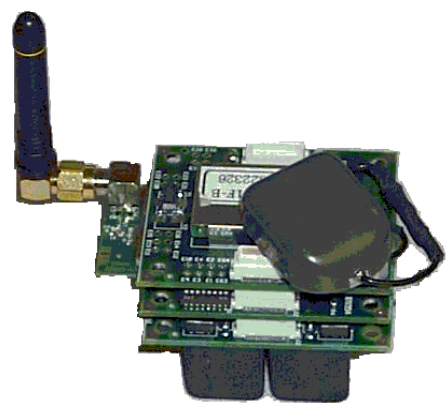

Figure 2: SDAC modules stacked to form a node.

Future developments of the platform will include further miniaturization, programming the modules to operate with more autonomy, implementation of more sensor modules, and integration of a high performance signal processing module.

The Data Fusion System (DFS) is the user interface to the SDAC system. The DFS collects the type and location of each node in the network and displays that information on a Geographical Information Systems (GIS) viewer that shows the location of nodes against geographical features such as roads, waterways, and terrain. Nodes designated to perform sensor fusion are shown larger and in green. Other nodes are shown in black. Figure 3 shows the GIS viewer and three sensor nodes. 


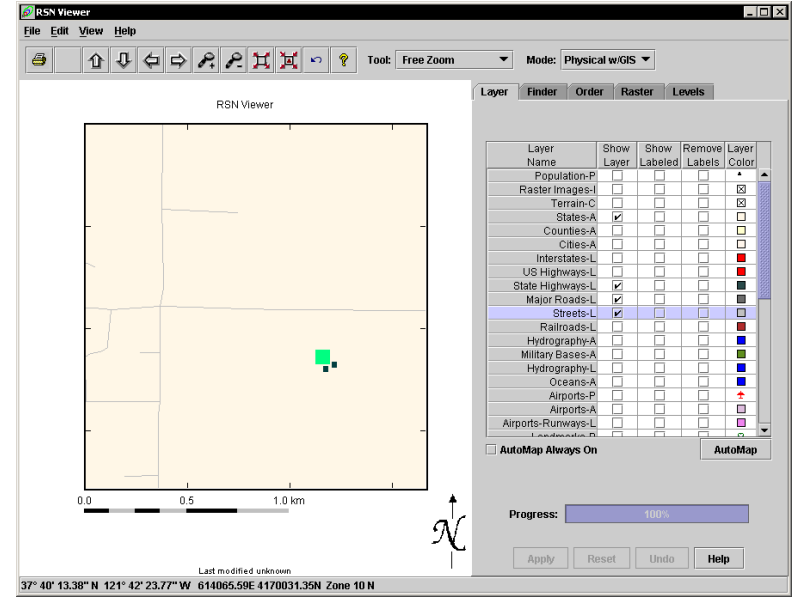

Figure 3: The GIS viewer showing a PIR, microphone, and geophone node and roads.

Events are displayed on the GIS viewer in real-time at the location they were detected. Blue icons on the GIS viewer indicate events generated by friendly objects, while red icons represent events generated by foreign objects. The icons persist on the GIS viewer as long as the object continues to generate events. The DFS stores events, containing the ID numbers of friendly objects that are present during an event, in a database. The DFS software can search the database and provide tracking information about friendly objects, which can then be rendered, on the GIS viewer to show the path of a friendly object as it moves through the network. Figure 4 shows a simulated network of 30 nodes tracking a friendly object as it moves through the area.

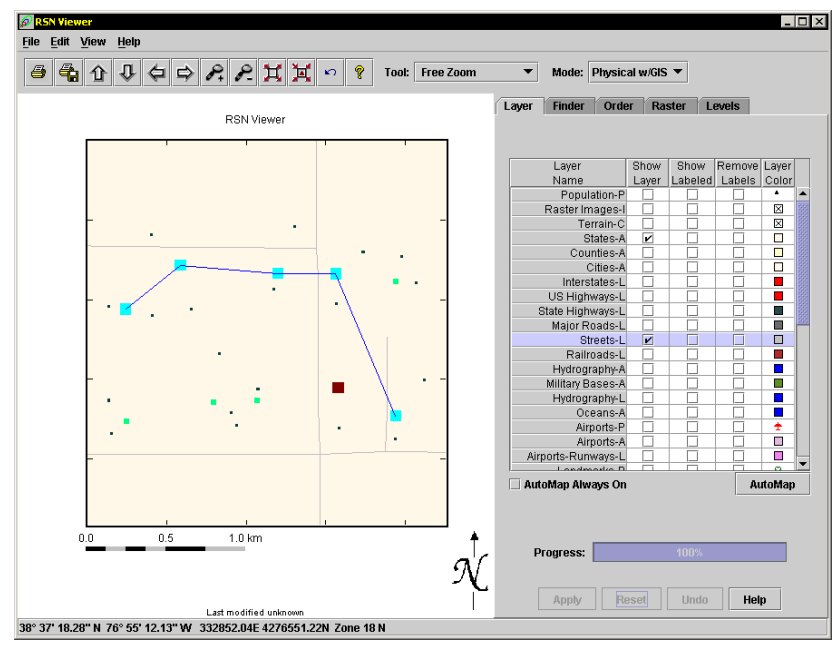

Figure 4: The GIS viewer showing a friendly object (light blue) being tracked through a simulated network and a single foreign object event (dark red).
The SDAC network consists of four types of nodes: microphone, geophone, PIR and transponder (PIR/T), and a gateway node that provides a connection to the DFS. Another part of the system, operating on a different frequency and independent of the network, is a reply transponder located on each friendly object. The system begins operation by establishing an ad-hoc wireless network among the nodes. Once established, the network detects sensor events and performs sensor fusion on the events. Since the PIR sensor has a finite range of only 10 to 30 feet, and the geophone and microphone sensors have ranges dependent on the magnitude of the events they are sensing, the PIR/T nodes were chosen as the sensor fusion nodes of the system. The PIR/T nodes store their own GPS-time stamped sensor events as well as receive wireless communications containing GPS-time stamped sensor events from neighboring microphone and geophone nodes. The PIR/T node applies sensor fusion rules to the events as they are received to determine if the sensor events constitute a verified event. A verified event is defined as a PIR event and a microphone event or a PIR event and a geophone event that occur within one second of each other. This fusion of two orthogonal phenomenona decreases the probability of false alarm in the detection of events. When a verified event occurs, the PIR/T node broadcasts an RF transponder query to its local area and collects friendly object replies from the object worn reply transponders. The time and location of the verified event, defined as the location of the PIR/T node and the time of the PIR event, and the ID numbers of the friendly objects that replied are packaged into a message and sent to the DFS, which displays the events in real-time on the GIS viewer.

Each type of node in the SDAC network contains a different compliment of modules. Microphone and geophone nodes contain a controller, a wireless networking module, a microphone or geophone sensor module, and a power supply module. The PIR/T node is similar to the microphone and geophone modules, but includes a PIR sensor module instead of a microphone or geophone sensor module and adds a transponder module. On start-up, the controller module performs an automatic discovery of the other modules present in its node. Once the controller has established which modules are present in the node, it automatically sets its own corresponding mode of operation so that the node can begin functioning appropriately.

The controller module serves as a central processor for the node. It receives events from sensor modules, determines if a verified event has occurred, sends query requests to the transponder module, receives query replies from transponder module, and operates a GPS receiver. 
The GPS receiver (a Furuno GN-80) is used to provide the controller with location data and to allow time synchronization among all the nodes in the network without the complication of a wireless communicationbased time synchronization protocol. The controller obtains the time from the GPS receiver and distributes time synchronization messages to the other modules in the node. This allows sensor events to be time-stamped on the sensor modules as soon as they occur. In order to maintain network-wide synchronization in the presence of clock drift, the controller modules obtain new time and location data from the GPS receiver every five minutes.

The networking module handles all aspects of the wireless communication in the network. It contains a 900 $\mathrm{MHz}$ radio and runs a low-power MAC layer called Sensor MAC (SMAC) developed at UCLA [10]. SMAC time-synchronizes nodes and allocates time slots to each node, thus allowing nodes to duty cycle their radios. This greatly reduces power consumption, but has the consequence of restricting when and how often nodes can transmit. SMAC uses a neighbor list to track the nodes with which it can communicate. This allows for simple neighbor-to-neighbor communication and broadcast and unicast functionality. Routed communication is accomplished with a lightweight proprietary routing algorithm. The networking module encapsulates all wireless networking functions. This keeps routed information from entering inter-module busses and reinforces the modularity of the system. Networking modules can also function as a gateway to the network for the DFS computer through a serial port and supporting hardware. The gateway node appears as a regular node to the rest of the network, but contains no other modules or sensors.

Sensor modules (the geophone, microphone, and PIR modules) consist of sensor circuitry and a microcontroller. The sensor circuitry is connected to the analog components onboard the microcontroller to create a levelsensitive trigger. The threshold for the trigger can be hardcoded or auto-calibrated by the microcontroller to the nominal output of the sensor circuitry. Whenever the sensor is triggered, the microcontroller generates a sensor event. The microcontroller time-stamps the event with its internal real time clock, and sends the event to its local controller module. If the node is a microphone or geophone node, the controller sends the event to the networking module, which in turn unicasts the message through its radio to its neighboring PIR/T nodes. If instead the node is a PIR/T node, the event is simply logged, and a check is performed to determine if any of the logged events create a verified event. Following a sensor event, the sensor module disables the comparator interrupt for a programmable period of time in order to de-bounce sensor operation. This results in a programmable maximum event frequency for each sensor module.

The transponder module is similar in functionality to a sensor module. The transponder module receives requests to broadcast a query from the controller and responds to the controller with a list of the friendly objects that reply to its broadcast. The reply list is empty if no friendly objects reply. The transponder module operates at $433 \mathrm{MHz}$, making its radio operation independent of the networking module. It broadcasts messages with a time and location stamp, and then listens for one second for replies from reply transponders. The replies contain the query time and location along with an appended friendly ID. The transponder validates each reply against the time and location of its most recent broadcast, and if the time and location match, the ID of the replying friendly is added to a growing list of friendly objects. When the one second long period elapses, the transponder packages the list of friendly objects and the time and location of the query into a message and sends the message to the controller.

The reply transponder resides on friendly objects in the field and listens for broadcasts from transponder modules. When the reply transponder receives a broadcast, it appends the friendly object's unique friendly ID to the original message and broadcasts this reply. The protocol and message structure used allow for future expansion of the information appended by the reply transponder. The system may thus eventually be used to collect information from friendly objects, such as physiological data or status, as they pass through the network.

The sensor, controller, and transponder modules were designed around a Cygnal C8051F124. The C8051F124 was chosen because of its large amount of RAM (8 kbytes), large amount of ROM (128 kbytes), onboard analog components (two comparators, two DACs, and an eight channel ADC), low power consumption (.5 $\mathrm{mA} / \mathrm{MHz}$ ), and high speed (up to $50 \mathrm{MHz}$ ). This combination of features provided a processor that could run a real-time operating system (RTOS) and was configurable to meet the needs of a variety of modules while offering substantial computing power and maintaining relatively low power consumption. The networking module was designed around a TI MSP430F149. The MSP430F149 is an ultra-low power RISC microprocessor. Since the MSP430F149 has only 2 kbytes of RAM, there is not enough memory to run an RTOS on top of the MAC layer and routing algorithm, and consequently, the networking module uses a software scheduler and relies heavily on interrupts and timers.

The controller, sensor, and transponder modules incorporate an RTOS into their firmware. Micro-C OS-II 
(uC/OS-II) was chosen as the RTOS because of its low cost, flexibility, availability for many processors, and scalability. uC/OS-II is a multithreaded preemptive RTOS with many features well suited to embedded systems. The common environment makes firmware development easier and more consistent among different modules. Using an RTOS has maintenance benefits since it makes adding new functionality as simple as adding a new task. Interfacing with existing tasks is as simple as posting to existing queues and semaphores. The RTOS also helps to avoid redesigning software from the ground up for new modules by providing an existing software platform.

\section{Results}

The proof-of-concept test system consisted of one geophone node, one microphone node, one PIR/T node, one reply transponder, one gateway node, and a laptop running the DFS software. In testing, the microphone node demonstrated the ability to detect loud voices at 10 $\mathrm{m}$, the geophone node was able to detect heavy footsteps at $5 \mathrm{~m}$, and the PIR node was able to detect a person at 10 $\mathrm{m}$. Acoustic and seismic events with larger magnitudes, such as vehicles passing by or loud claps could be detected at much greater distances. While the networking module radio can in theory transmit $300 \mathrm{~m}$ line-of-sight, it was limited to less than $100 \mathrm{~m}$ in practice. These facts combined to allow the system as built to detect people and vehicles as they passed through an area about $100 \mathrm{~m}^{2}$ in size. In theory, the system could be expanded to include nearly $2^{16}$ nodes and cover hundreds of square kilometers. In practice, however, the system is most likely limited to an area a few square kilometers in size due to the networking and routing protocols used and the necessary density of the range-limited sensors.

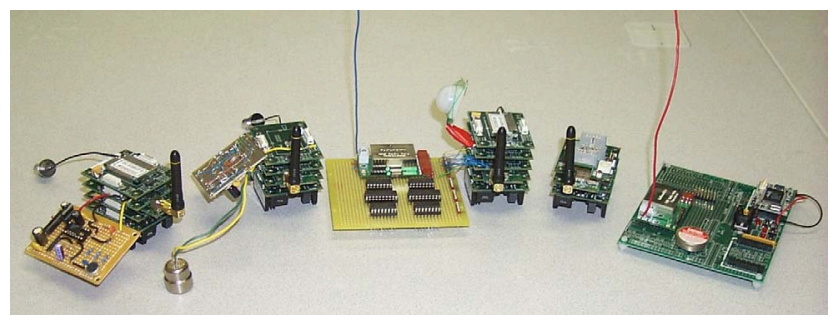

Figure 5: Left to right: microphone, geophone, PIR/T, and gateway nodes and the reply transponder.

Of the many difficulties encountered in developing this system, debugging the hardware and firmware in each module and providing adequate power isolation to each module were the most difficult to solve. Since the modules share a common bus, a common programming interface was designed into each module. Because of this, only one module can be debugged in any node at a time. To solve this problem, an expansion board is being developed that will allow the modules to be connected together by a ribbon cable as if they were stacked, but without sharing a common programming interface. Additionally, stacking the modules makes probing the hardware during operation difficult. The expansion board will facilitate debugging of the modules while they operate as part of a node and allow easier access to the hardware for probing.

Power isolation proved to be the most surprising problem with system. Even though the power supply module provides adequate isolation from the DC/DC converters, voltage spikes and ripple were able to move from one module to another through their common power lines. Actions such as turning the GPS receiver on and off, turning the radio on and off, and changing processor clock speeds introduced large voltage spikes into the power rails that in some cases interfered with sensor circuitry operation. To solve this problem, LC power filters were added to the networking modules and larger input capacitors were added to the controller and sensor modules. An additional low-dropout regulator was used to further isolate the sensor circuitry from the rest of the system.

\section{Conclusion}

The SDAC system provides a way to detect and localize objects moving through an area, distinguish friendly objects from foreign objects, and track friendly objects. The system demonstrates that currently available technology can be used to implement a fully functional sensor network capable of performing in-network sensor fusion. The sensor fusion demonstrates a method of distributed localization of friendly and foreign objects. The data reduction performed by the network allows the end-user software to perform friendly object tracking by simply searching a database. The modular architecture developed to create the system allows for a more extensible and upgradeable system than standard centralized systems. More research is needed to refine the system and further develop the modular architecture. In particular, careful attention needs to be paid to providing methods for debugging the system and isolating the power supplies of individual modules from interference caused by the rest of the node. Innovations in sensor technology, wireless communications, power-aware software techniques, and distributed computing will continue to drive future developments of advanced sensor networks like SDAC. 


\section{References}

[1] V. Raghunathan, C. Schurgers, S. Park, and M. Strivastava, "Energy-Aware Wireless Microsensor Networks," IEEE Signal Processing, IEEE, March, 2002, pp. 40-50.

[2] S. S. Intille, K. Larson, "Designing and Evaluating Supportive Technology for Homes," Proceedings of the IEEE/ASME International Conference on Advanced Intelligent Mechatronics 2003, IEEE, Port Island, Kobe, Japan, July, 2003.

[3] A Stroupe, M. Martin, and T. Balch, "Distributed Sensor Fusion for Object Position Estimation by Multi-robot Systems," Proceedings of the IEEE Conference on Robotics and Automation, Seoul, Korea, 2001, pp. 1092--1098.

[4] L. Chen and N. Tokuda, "Multitarget Tracking By Distributed Cooperative Processing", In: M. Syed, O.Baiocchi and G.Lasker eds: Advances in Intelligent Computing and Multimedia Systems (Proceedings of International Symposium on Intelligent Multimedia and Distance Education, Baden Baden, Germany, August 2-7, 1999), The International Institute for Advanced Studies in Systems Research and Cybernetics, University of Windsor, Canada, 1999, pp.217--222.

[5] B. Jung, and G. S. Sukhatme, "A Region Based Approach for Cooperative Multi-target Tracking in a Structured Environment", IEEE/RSJ International Conference on Intelligent Robots and Systems, 2002, EPFL, Switzerland, September, 2002, pp. 2764-2769.

[6] Norihiko Yoshida, "Multi-Target Tracking by Cooperation of Stationary and Mobile Agents", 4th International Workshop on Cooperative Distributed Vision, Kyoto, Japan, March, 2001, pp. 183-203.

[7] B. Horling, R. Vincent, R. Mailler, J. Shen, R. Becker, K. Rawlins, and V. Lesser, "Distributed Sensor Network for Real Time Tracking," Proceedings of the Fifth International Conference on Autonomous Agents, ACM Press, 2001, pp. 417-424.

[8] J. Davis, R. Kyker, N. Berry, A System Level Hardware Architecture for a Distributed Sensor Network Node, Sandia National Labs, Livermore, California, February, 2003.

[9] J. Davis, R. Kyker, N. Berry, "Description and Analysis of a Flexible Hardware Architecture for Event-Driven Distributed Sensor Network Nodes", International Telemetry Conference, October, 2003.

[10] W. Ye, J. Heidemann, D. Estrin, "An Energy-Efficient MAC Protocol for Wireless Sensor Networks," Proceedings of the 21st International Annual Joint Conference of the IEEE Computer and Communications Societies (INFOCOM 2002), IEEE, New York, NY, USA, June, 2002. 